\section{Erythromycin therapy for cryptosporidiosis?}

\section{To the Editor:}

In the article, "Bronchial and gastrointestinal cryptosporidiosis in AIDS" (JAOA 1989;89:195-197), Dr Goodstein and colleagues state that the patient improved after receiving erythromycin therapy. The authors conclude this report by citing literature that they claim supports the use of this agent in the treatment of cryptosporidiosis.

However, the recent literature ${ }^{1,2}$ does not support their claim. Spiramycin is the only antibiotic that has demonstrated modest efficacy with only minimal toxicity when used to treat this infection. ${ }^{1,3}$

Furthermore, as the case report is presented, it is not possible to ascertain whether other pulmonary pathogens were adequately excluded. Similarly, whether the diarrhea relented spontaneously or in response to the erythromycin remains unclear.

While it is possible that erythromycin or other agents may be effective in treating cryptosporidiosis, the only way to determine this is through appropriate prospective trials. Generalizations based on indi- vidual case findings - "last case" phenomenon-prove dangerous for both patient care and education. ${ }^{4}$ The use of erythromycin to treat cryptosporidiosis should not be recommended based on this single report.

JOHN A. SELLICK, JR, DO

Clinical Assistant Professor of Medicine

Department of Medicine

Division of Infectious Disease

State University of New York at Buffalo

1. Soave R: Cryptosporidiosis and isosporiasis in patients with AIDS. Infect Dis Clin North Am 1988;2:485-493.

2. Update: Treatment of cryptosporidiosis in patients with acquired immunodeficiency syndrome (AIDS). MMWR 1984;33:117-119.

3. Portnoy D, Whiteside ME, Buckley E, MacLeod CL: Treatment of intestinal cryptosporidiosis with spiramycin. Ann Intern Med 1984;101:202-204.

4. Waldman RH: The role of epidemiology in medical education. Infect Control 1987;8:9596.

\section{Response}

For the sake of brevity, the results of tests performed to exclude pulmonary pathogens were not detailed. However, they did include washings, brushings, and bronchoscopy biop- sies, all of which were negative for typical and atypical acid-fast bacilli and Pneumocystis carinii; and routine cultures and sensitivity studies for fungi, Coccidioides immitis, Cryptococcus neoformans, and cytomegalovirus.

Likewise, considering that diarrhea persisted for 1 week prior to erythromycin therapy but resolved completely within 48 to 72 hours posttreatment, it is unlikely this resolution occurred spontaneously.

We do agree that a single case does not constitute a therapeutic basis for other patients' regimens. My colleagues and I merely purposed to report the salutary effects of erythromycin therapy in a patient with documented presence of bronchial and gastrointestinal Cryptosporidium. As noted in our summary, "In these cases [of Cryptosporidium pneumonitis], erythromycin has been used with symptomatic improvement. No long-term studies on microbiologic cure are available." The article merely suggests that, as Dr Sellick points out, erythromycin therapy may be effective in the treatment of cryptosporidiosis.

RICHARD S. GOODSTEIN, DO York, $\mathrm{Pa}$ 


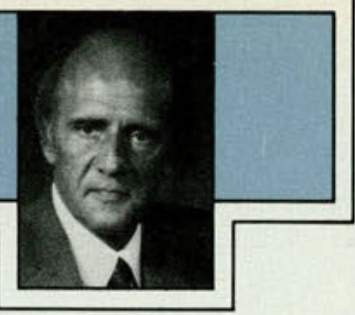

\section{Home IV Antibiotic Therapy: An Introduction}

Lowering health care costs while maintaining high quality care is a goal that all physicians strive for. An important step toward this goal is the development of home health care that is both therapeutically and economically effective. One particular aspect of home health care that is gaining acceptance is home intravenous antibiotic therapy.

\section{Evolution of an Industry}

Experts anticipate that over the next several years home IV antibiotic therapy will be one of the fastest growing segments of the home health care market. Today, physicians have access to an industry that provides necessary technology, personal services and pharmaceutical supplies - an industry devoted to home health care. With the growth of this industry, most physicians will be able to successfully manage many patients with a minimum of acute hospital care.

\section{The Home IV Therapy Decision}

Almost any patient who requires IV antibiotic therapy, and is hospitalized to receive that therapy, could be considered for treatment at home. However, four criteria are often applied before implementing home IV therapy: ${ }^{1}$

1. No suitable oral therapy is available.

2. The patient's condition is stable enough for discharge, and the patient can monitor his/her own therapy.

3. The patient agrees to outpatient therapy after a full disclosure of responsibilities and potential problems.

4. The patient has a suitable home environment, including a phone for emergency communications and a refrigerator for antibiotic storage.

Any infection that responds to IV therapy can potentially be treated at home, including osteomyelitis, endocarditis, wound infections, urinary tract infections, septic arthritis and others. ${ }^{2}$

Many classes of antibiotics appear suitable for home IV infusion; however, antibiotics used in outpatient care should have low toxicity, a broad spectrum of activity and a long half-life, allowing for less frequent dosing. Antibiotics with long half-lives enhance the convenience and cost effectiveness of home therapy and facilitate patient compliance.

\section{Advantages}

Home IV antibiotic therapy removes the risk of nosocomial infection. Receiving therapy at home in warm and familiar surroundings also provides psychological benefits. Another benefit is that patients in the home care environment become more active in their recovery. These intangibles appear to contribute to more rapid and predictable recovery.

There are also considerable financial advantages to home IV antibiotic therapy. For those patients who are able to work while receiving therapy, the benefits are obvious. Of course, savings are also accrued because patients are not hospitalized for long periods of time. In one study, the calculated savings ranged from $\$ 2,791$ to $\$ 4,651$ per patient. $^{3}$

In addition, reimbursement for home services has greatly improved during the past five years. Many insurers now pay for outpatient therapy that can replace treatment given in the hospital. Congress has also provided for such reimbursement in the Medicare population under the catastrophic health insurance law that was recently enacted.

The success of home IV antibiotic therapy depends on a team effort employing sound case management by the physician and quality service from the home health care industry. Teamwork by these two groups will help ease the medical profession into a new era of health care.

\section{References}

1. Larson SE: Home IV antibiotic therapy: The primary-care physician's role. Drug Therapy, Nov 1987:67-74.

2. Rising JB: Home IV antibiotic therapy: Roles you can play. U.S. Pharmacist, Oct 1987:H9-H14.

3. Graves G, Jackson JP, Maxwell A, Woods T: Home intravenous antibiotic therapy in Arkansas. J Arkansas Med Soc 1987;84:55-57. 